\title{
Cytotoxic mechanism of PLK1 inhibitor GSK461364 against osteosarcoma: Mitotic arrest, apoptosis, cellular senescence, and synergistic effect with paclitaxel
}

\author{
YI-SHENG CHOU ${ }^{1,2,6-8}$, CHUEH-CHUAN YEN ${ }^{1-3,6}$, WEI-MING CHEN ${ }^{3-6}$, \\ YUNG-CHAN LIN ${ }^{1-3}$, YAO-SHAN WEN ${ }^{1-3}$, WEI-TING KE ${ }^{1-3}$, JIR-YOU WANG $^{3,4}$, CHUN-YU LIU ${ }^{1-3,6}$, \\ MUH-HWA YANG ${ }^{1,2,6,7}$, TAIN-HSIUNG CHEN ${ }^{3,4,6}$ and CHIEN-LIN LIU ${ }^{3,4,6}$
}

\begin{abstract}
${ }^{1}$ Division of Medical Oncology, Department of Oncology, ${ }^{2}$ Division of Hematology and Oncology, Department of Medicine, ${ }^{3}$ Therapeutical and Research Center of Musculoskeletal Tumor, ${ }^{4}$ Department of Orthopedics and Traumatology, ${ }^{5}$ The Rehabilitation and Technical Aid Center, Taipei Veterans General Hospital, Taipei;

${ }^{6}$ School of Medicine, ${ }^{7}$ Institute of Clinical Medicine, National Yang-Ming University, Taipei; ${ }^{8}$ Division of Hematology and Oncology, Department of Medicine, Lo-Hsu Foundation, Lotung Poh-Ai Hospital, Luodong, Yilan, Taiwan, R.O.C.
\end{abstract}

Received November 24, 2015; Accepted December 19, 2015

DOI: 10.3892/ijo.2016.3352

\begin{abstract}
Polo-like kinase 1 (PLK1), a serine/threonine kinase and an oncogene, is crucial in regulating cell cycle progression. PLK1 also has been demonstrated as a potential target of osteosarcoma (OS) by using short hairpin RNA libraries in lentiviral vectors for screening of protein kinase. In preclinical studies, GSK461364, a potent and selective ATP-competitive PLK1 inhibitor, showed antiproliferative activity against multiple tumor cell lines. In the present study, we evaluated the expression level of PLK1 in OS and explored the cytotoxic mechanism of GSK461364 against OS. PLK1 was significantly overexpressed in OS compared with normal osteoblasts and other types of sarcoma. GSK461364 inhibited PLK1 and caused mitotic arrest by inducing G2/M arrest in OS cells. Moreover, GSK461364 exerted a cytotoxic effect by inducing apoptosis in OS, and induced cellular senescence in OS cell lines, as indicated by an increased senescenceassociated $\beta$-galactosidase activity and enhanced DcR2 and interleukin-1 $\alpha$ expression. In addition, we demonstrated a synergistic cytotoxic effect of GSK461364 and paclitaxel, possibly resulting from combined mitotic arrest. In conclusion, the present study revealed that PLK1 was overexpressed in OS and that GSK461364 exerted its cytotoxic effect on OS by inducing mitotic arrest and subsequent apoptosis and induced cellular senescence; therefore, senescence-associated markers
\end{abstract}

Correspondence to: Dr Chueh-Chuan Yen, Division of Medical Oncology, Department of Oncology, Taipei Veterans General Hospital, No. 201, Sec 2, Shih-Pai Road, Taipei 112, Taiwan, R.O.C.

E-mail: ccyen@vghtpe.gov.tw

Key words: cellular senescence, GSK461364, osteosarcoma, paclitaxel, polo-like kinase 1 can be used as treatment biomarkers, and a combination of GSK461364 and paclitaxel can potentially treat OS.

\section{Introduction}

Osteosarcoma (OS) is the major subtype of malignant bone tumors $(1,2)$. In pediatric patients with localized disease, $\sim 80-90 \%$ of 5-year overall survival is achieved when treated at experienced centers (3-5); however, in adult patients $(6,7)$ and patients with recurrent or metastatic disease $(3,8,9)$, response to chemotherapy is less satisfactory, and survival of such patients is generally poor.

Genetically, sarcomas can be classified as translocationrelated and non-translocation-related. Non-translocationrelated sarcomas can be further grouped as sarcomas with a simple genetic profile based on limited amplifications, such as dedifferentiated liposarcomas and parosteal OS $(10,11)$, and major sarcomas with extremely complex genomic imbalances, such as leiomyosarcomas, undifferentiated pleomorphic sarcomas, pleomorphic liposarcomas and conventional OS (12). In conventional OS, these genomic aberrations cause oncogenic changes in such diverse processes as cell cycle regulation, cell death/cytokine pathways, proliferative signaling pathways, telomere dysfunction, metastasis and tumor suppression (13-20). Recently, several studies have revealed that the PI3K-mTOR pathway is crucial in conventional OS $(15,16)$. However, the result of a clinical study of the mTOR inhibitor in OS treatment was unsatisfactory (21). Therefore, additional investigations exploring potential OS targets are necessary.

Polo-like kinase 1 (PLK1), a serine/threonine kinase and a known oncogene, is crucial in regulating cell cycle progression (22). Moreover, PLK1 has been demonstrated to be a potential target of OS when short hairpin RNA libraries were used in lentiviral vectors for protein kinase screening $(23,24)$. However, few studies have evaluated PLK1 expression in 
OS (25). In our previous study, we revealed that 15-deoxy- $\Delta 12$, 14-prostaglandin J2 (15d-PGJ2), a prostaglandin derivative, exerts cytotoxic effects against OS cells by downregulating the p-AKT and PKA-PLK1 pathways through reactive oxygen species-mediated c-Jun-N-terminal kinase activation (26). Therefore, inhibiting PLK1 may effectively treat OS.

GSK461364, a potent and selective ATP-competitive PLK1 inhibitor, has exhibited antiproliferative activity against multiple tumor cell lines in preclinical studies (27). Elevation of phosphorylated histone $\mathrm{H} 3$ (pHisH3) and suppression of PLK1 were indicators observed in tumor xenografts (27). In the present study, we evaluated the expression level of PLK1 in OS and explored the cytotoxic mechanism of GSK461364 against OS.

\section{Materials and methods}

Bioinformatics analysis. In total, 109 microarray and clinicopathological data sets of sarcoma from Gene Expression Omnibus (GEO) dataset GSE14827 (OS), GSE13433 (alveolar soft part sarcoma, ASPS), GSE8167 (gastrointestinal stromal tumor, GIST), GSE20196 (synovial sarcoma, SYN), and GSE20559 (liposarcoma, LPS) and from E-MEXP-1922 (leiomyosarcoma, LMS) were obtained from the NCBI and ArrayExpress websites, respectively. Nine microarray data sets of normal osteoblasts (GSE9451 and GSE10311) were retrieved from the NCBI website. All these datasets were in Affymetrix U133 Plus 2.0 platform. To decrease intrasubtype heterogeneity, not all retrieved samples were included in the analysis. For OS, only tumors without subsequent metastasis were selected; for GIST, only those with exon 11 mutation were included; for SYN, only tumor with SYT-SXX type 1 fusion gene and non-poorly differentiated histology were put into analysis; and for LPS, only well-differentiated (WD) and dedifferentiated (DD) tumors were chosen due to their similar genetic background. Gene expression data were normalized using dChip $(28,29)$. The expression levels of individual genes were obtained through Z-score transformation, and the differences between different subtypes were subsequently compared using the Student's t-test.

Cell lines and reagent. Three OS cell lines, U2OS, MG63 and SJSA were selected for in vitro study and maintained in either Dulbecco's modified Eagle's medium (DMEM), Iscove's modified Dulbecco's medium, or DMEM/F12 base media with $10 \%$ fetal bovine serum. GSK461364 was purchased from Calbiochem (San Diego, CA, USA). The following antibodies were used for immunoblotting: PLK1 (\#4513; 1:1,000), phospho-cdc25C (Ser216) (pCDC25C) (\#4901; 1:1,000), phospho-Histone H3 (Ser10) (pHisH3) (\#9701; 1:1,000), and phospho-Histone H2A.X (Ser139) (pH2AX) (\#2577; 1:1,000), all from Cell Signaling Technology, and anti-actin (ABS 24-100; $1: 10,000)$.

Cell viability assay. Two methods were used in the cell viability assay, the first being the TACS ${ }^{\mathrm{TM}}$ 3-(4,5-dimethylthiazol-2-yl)2,5-diphenyltetrazolium bromide (MTT) cell proliferation assay (Trevigen, Gaithersburg, MD, USA). Approximately 2,000-20,000 cells/100 $\mu \mathrm{l} /$ well were seeded in 96-well plates overnight. Subsequently, reagents at different concentrations were added in triplicates. The plates were incubated for the desired time at $37^{\circ} \mathrm{C}$, pulsed with $10 \mu 1$ MTT reagent, and incubated for an additional $4 \mathrm{~h}$ at $37^{\circ} \mathrm{C}$. Furthermore, detergent reagent of $100 \mu \mathrm{l} /$ well was added and mixed thoroughly to dissolve the blue crystals. Absorbance of the converted dye was measured using a Vmax microplate reader (Molecular Devices, Sunnyvale, CA, USA) at $570 \mathrm{~nm}$ (test) and $650 \mathrm{~nm}$ wavelengths. Cell survival was calculated using the following equation: \% survival $=($ mean experimental absorbance $/$ mean control absorbance) x 100 (30).

The possible synergistic effect of GSK461364 and chemotherapy was measured using the combination index (CI) calculated using CalcuSyn software (Biosoft, Ferguson, MO, USA) (31). CI $>1$ was defined as antagonism, $\mathrm{CI}=1$ as additivity, and $\mathrm{CI}<1$ as synergy; the experiment was performed in triplicate.

Cell viability was also measured through a trypan blue exclusion assay (32). OS cells were plated at $5 \times 10^{4}$ cells/well in 24-well plates; a variable concentration of GSK461364 was added to each well the following day, and the plates were incubated at $37^{\circ} \mathrm{C}$ for $72 \mathrm{~h}$. Subsequently, the cells were trypsinized and mixed with trypan blue. Viable cells with intact cell membranes and not absorbing trypan blue were counted using a light microscope. All experiments were performed in triplicate.

Apoptosis assessment by Annexin V staining. Annexin V staining was used to detect apoptosis in cell lines treated with GSK361364. The cells were washed once with $1 \mathrm{X}$ phosphate-buffered saline (PBS) following drug treatment and resuspended in $100 \mu$ l staining solution [containing Annexin V fluorescein and propidium iodide in an Annexin V-binding buffer, Annexin V-fluorescein isothiocyanate (FITC); BD Biosciences, San Diego, CA, USA]. Subsequently, the cells were incubated at room temperature for $15 \mathrm{~min}$ and diluted in 1X Annexin V-binding buffer. The percentages of apoptotic cells were measured through flow cytometry.

Cell cycle analysis. Cell cycle analysis was performed through flow cytometry, as previously described (33). Briefly, cells were trypsinized, washed twice with PBS, and fixed with $70 \%$ ethanol at $-20^{\circ} \mathrm{C}$ overnight, following which the fixed cells were washed twice with cold PBS, suspended in $420 \mu \mathrm{l}$ PBS, added with $50 \mu 110 \mathrm{mg} / \mathrm{ml}$ RNase A (Sigma), and shaken at $37^{\circ} \mathrm{C}$ for $15 \mathrm{~min}$. Subsequently, $20 \mu \mathrm{l}$ of $0.2 \mathrm{mg} / \mathrm{ml}$ propidium iodide (PI) was added and cells were retained at room temperature for $1 \mathrm{~h}$. Flow cytometry was performed using FACSCalibur (Becton-Dickinson and Co., Oxford, CA, USA) to measure relative DNA content based on red fluorescence levels. The percentages of the cells in the different phases of the mitotic cell cycle were calculated using CellQuest software (Becton-Dickinson).

Western blotting. Monolayers of cultured cells were rinsed in PBS and scraped into lysis buffer [ $25 \mathrm{mM}$ Tris- $\mathrm{HCl} \mathrm{pH} 7.6$, $150 \mathrm{mM} \mathrm{NaCl}, 1 \% \mathrm{NP}-40,1 \%$ sodium deoxycholate, $0.1 \%$ SDS (Thermo Fisher Scientific)] containing protease and phosphatase inhibitor cocktail (1:100 dilution; Thermo Fisher Scientific). Lysates were incubated for $30 \mathrm{~min}$ at $4^{\circ} \mathrm{C}$ and subsequently clarified through centrifugation for $30 \mathrm{~min}$ at 
$13,200 \mathrm{rpm}$ at $4^{\circ} \mathrm{C}$. Protein concentrations were determined with the Pierce BCA protein assay kit (Thermo Fisher Scientific). Protein extracts (20-50 $\mu \mathrm{g} /$ lane) were electrophoretically separated on sodium dodecyl sulfate-polyacrylamide gel electrophoresis gels, transferred to polyvinyl difluoride membranes (Millipore), and blotted with specific antibodies. Immunoreactive bands were detected using enhanced chemiluminescence (Millipore) and exposed to X-ray film.

Senescence-associated $\beta$-galactosidase assay. Senescenceassociated $\beta$-galactosidase (SA- $\beta$-gal) activity was detected using the cellular senescence assay kit (Millipore) according to the manufacturer's instructions. U2OS cells were treated with GSK461364 for $72 \mathrm{~h}$; the adherent cells were fixed and stained with X-gal in a staining solution at $\mathrm{pH} 6.0$ and washed twice with $1 \mathrm{X}$ PBS. The percentage of SA- $\beta$-gal-positive cells (the number of positive cells relative to the total number of cells) was quantified by counting 100 cells in 3 randomly chosen fields per dish by using an Olympus IX51.

DcR 2 expression assayed by flow cytometry. DcR 2 expression was detected through flow cytometry. OS cells treated with GSK461364 for $72 \mathrm{~h}$ were washed twice with 1X PBS and incubated with Alexa Fluor 488-labeled anti-DcR2 (R\&D Systems, Minneapolis, MN, USA) for $30 \mathrm{~min}$ at room temperature in the dark. After washing twice with $1 \mathrm{X}$ PBS and resuspending in $1 \mathrm{X}$ PBS, the mean of fluorescence intensity on the cell surface was measured through flow cytometry using FACSCalibur (Becton-Dickinson).

Analysis of interleukin-1 $\alpha$ expression. RNAs were extracted from the cell lines using TRIzol ${ }^{\circledR}$ reagent (Invitrogen) according to the manufacturer's instructions and reverse transcribed with $1 \mu \mathrm{g}$ RNA by using SuperScript ${ }^{\circledR}$ III First-Strand Synthesis system (Invitrogen) for reverse transcription polymerase chain reaction (RT-PCR). The copy number for both interleukin (IL)-1 $\alpha$ and glyceraldehyde 3-phosphate dehydrogenase (GAPDH) mRNA was measured through qRT-PCR by using Maxima SYBR-Green/ROX qPCR Master Mix (Thermo Fisher Scientific) and a LightCycler ${ }^{\circledR} 480$ system (Roche). The primer sequences used in the reaction were as follows: IL-1 $\alpha$ forward, 5'-CCGTGAGTTTCCCAGAAGAA-3' and IL-1 $\alpha$ reverse, 5'-ACTGCCCAAGATGAAGACCA-3'; GAPDH forward, 5'-GCCAAGGTCATCCATGACAACT-3' and GAPDH reverse, 5-GAGGGGCCATCCACAGTCTT-3' (34). The PCR cycling conditions were as follows: $95^{\circ} \mathrm{C}$ for $5 \mathrm{~min}$ followed by 45 cycles of $95^{\circ} \mathrm{C}$ for $30 \mathrm{sec}, 55^{\circ} \mathrm{C}$ for $30 \mathrm{sec}$ followed by $72^{\circ} \mathrm{C}$ for $40 \mathrm{sec}$. The gene expression levels were calculated as previously described (35).

\section{Results}

Overexpression of PLK1 in OS. We explored the expression level of PLK1 among normal osteoblasts and OS as well as among other types of sarcoma. The expression level of PLK1 was compared between 27 OS and 9 normal osteoblasts. As depicted in Fig. 1A, PLK1 was significantly overexpressed in OS compared with normal osteoblasts. We further compared the expression of PLK1 in OS with other types of sarcoma. As depicted in Fig. 1B, the transcript level of PLK1 was signifi-
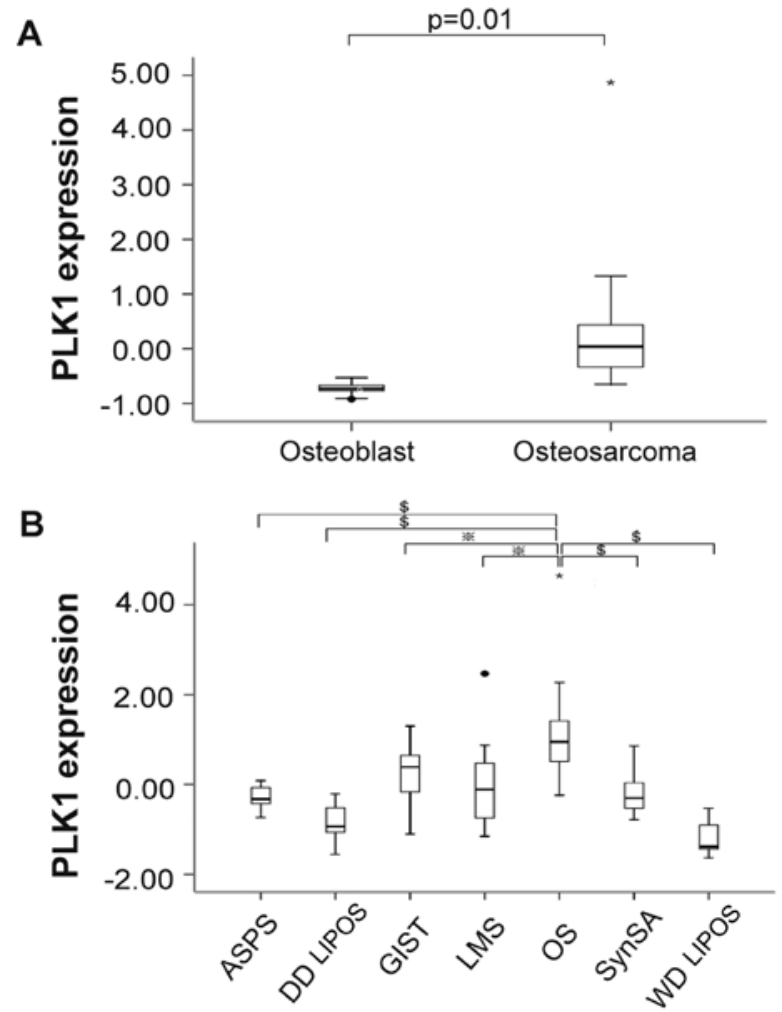

Figure 1. Comparing the expression level of PLK1 (A) between osteoblast and osteosarcoma, (B) between different types of sarcoma. Osteosarcoma showed significant higher expression level than either osteoblast or other types of sarcoma. ASPS, alveolar soft part sarcoma; DD LipoS, dedifferentiated liposarcoma; GIST, gastrointestinal stromal tumor; LMS, leiomyosarcoma; OS, osteosarcoma; SYN, synovial sarcoma; WD LipoS, well-differentiated liposarcoma. ${ }^{*} \mathrm{P}<0.01 ;{ }^{\$} \mathrm{P}<0.001$.

cantly higher in OS compared with other types of sarcoma. These results indicated that PLK1 was overexpressed in OS.

GSK461364-mediated PLK1 inhibition and mitotic arrest in OS cell lines. An in vitro model was used to explore the potential of PLK1 as a therapeutic target in OS. GSK461364, a potent PLK1 inhibitor, has demonstrated favorable activity in various types of cancers (27). OS cells were treated with GSK461364 and the levels of PLK1 and pCDC25C (a downstream effector of PLK1) were measured through western blotting. Except for U2OS in time-dependent studies, decreased level of PLK1 and pCDC25C were noted in all three GSK461364-treated OS cell lines (Fig. 2). Moreover, we demonstrated that all OS cell lines treated with GSK461364 exhibited a dose- and time-dependent increase in pHisH3, an indicator of mitotic arrest (Fig. 2).

Effects of GSK461364 on cell cycle progression in OS cell lines. Furthermore, we explored the effects of GSK461364 on cell cycle progression in OS cell lines. As depicted in Fig. 3, flow cytometric analysis of DNA content in cells treated with GSK461364 for $24 \mathrm{~h}$ demonstrated marked accumulation of cells at G2/M DNA content in all 3 OS cell lines.

Cell viability assays were used to detect the possible cytotoxic activity of GSK461364 against OS cell lines. As depicted in Fig. 4, GSK461364 displayed cytotoxicity against all the three OS cell lines when assessed using either an MTT assay (Fig. 4A), or a trypan blue exclusion assay (Fig. 4B). Moreover, 


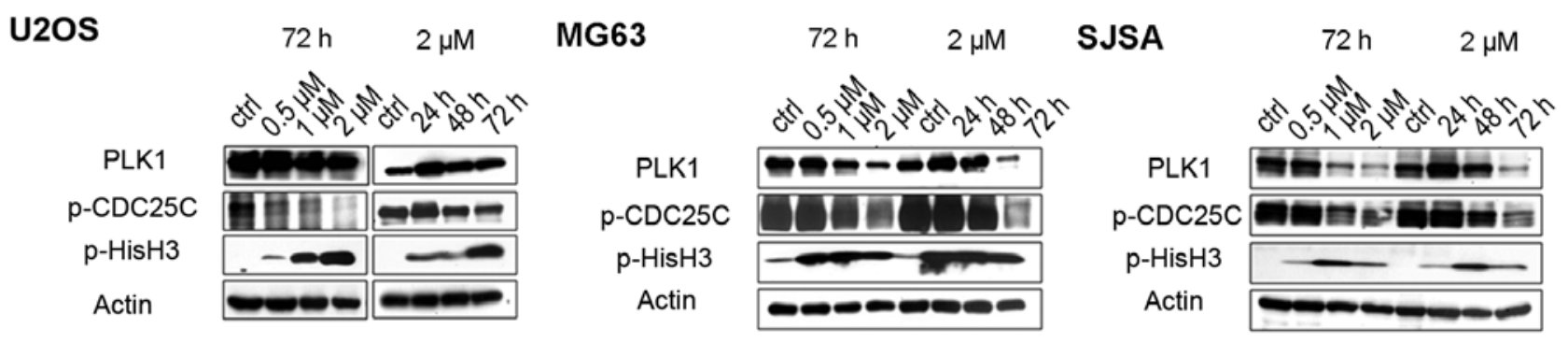

Figure 2. Immunoblotting with antibodies against PLK1, phosphorylated CDC25C (pCDC25C), phosphorylated histone $\mathrm{H} 3$ (pHisH3), and actin in three osteosarcoma cell lines after being treated with GSK461364. Except for U2OS in time-dependent studies, decreased level of PLK1 and pCDC25C were noted in the three GSK461364-treated OS cells. Also a dose-dependent and time-dependent increase in pHisH3 was observed the GSK461364-treated OS cells

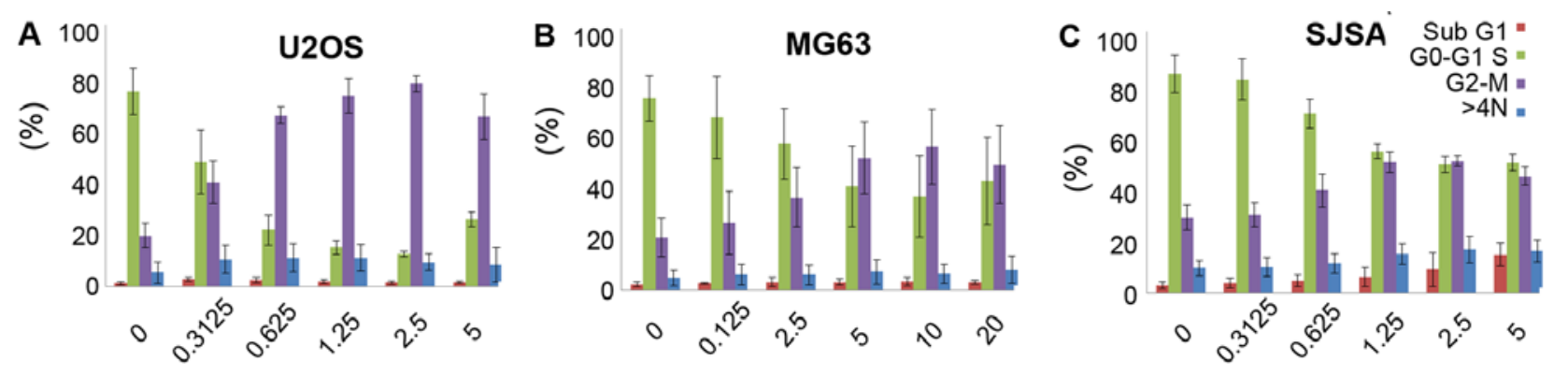

Figure 3. GSK461364 induces G2/M arrest in osteosarcoma cell lines. The DNA profiles of (A) U2OS, (B) MG63 and (C) SJSA treated with dimethyl sulfoxide (DMSO) or GSK461364 for $24 \mathrm{~h}$ were evaluated by flow cytometry. The percentages of cells in the sub G1, G0/G1, S and G2/M phases, as well as the percentage of cells with a DNA content $>4 \mathrm{~N}$ are shown in bars with different colors.
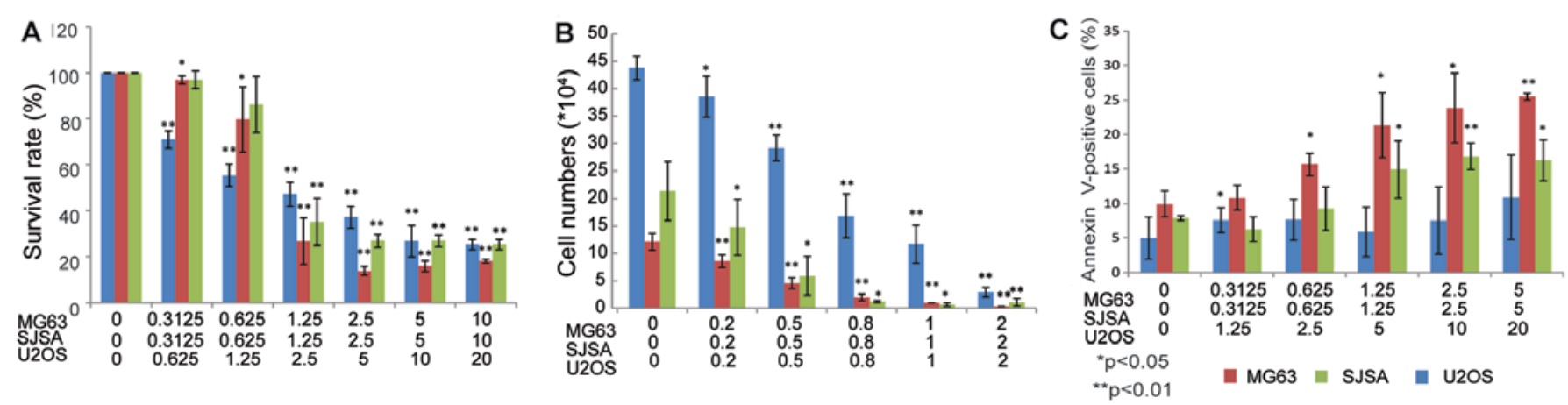

Figure 4. Treatment with GSK461364 inhibits PLK1 and results in growth inhibition and apoptosis in osteosarcoma cell lines. The viability of U2OS, MG63 and SJSA cells after treatment with various concentration of GSK461364 for $72 \mathrm{~h}$ was measured with (A) the TACS ${ }^{\mathrm{TM}}$ MTT cell proliferation assay (expressed as a percentage of viability under controlled culture conditions) and (B) a trypan blue exclusion assay (expressed as viable cell number). (C) Apoptosis assay. Three osteosarcoma cell lines were treated with various concentration of GSK461364 for $72 \mathrm{~h}$. The percentage of apoptotic cells was determined using Annexin V-FITC/PI staining. All data represent the mean $\pm \mathrm{SD}$ of three independent experiments. ${ }^{*} \mathrm{P}<0.05 ;{ }^{* *} \mathrm{P}<0.01$.
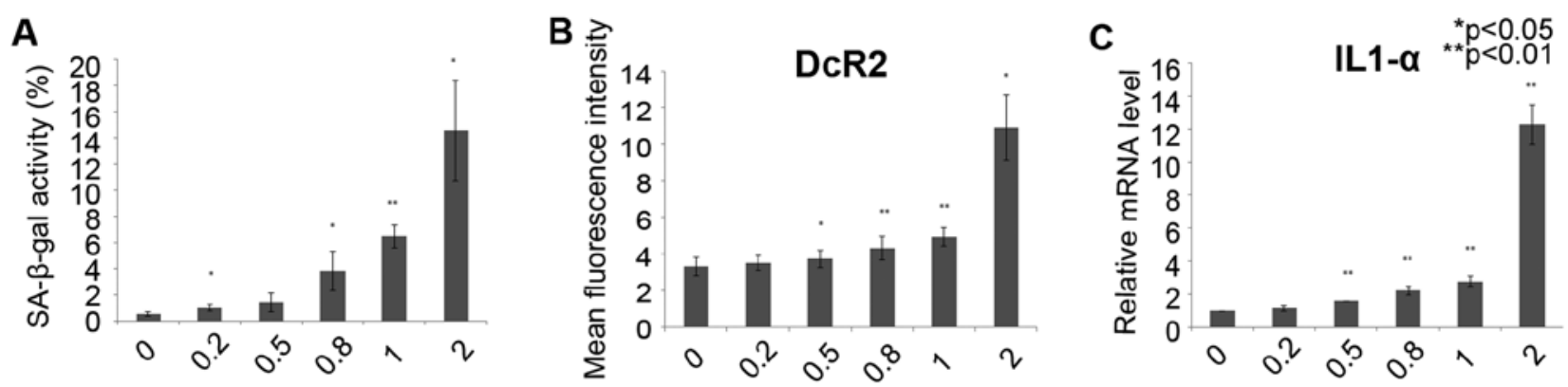

Figure 5. GSK461364 treatment induces cellular senescence in U2OS cell lines. (A) Treating U2OS cells with GSK461364 for $72 \mathrm{~h}$ dose-dependently increased senescence-associated $\beta$-galactosidase (SA- $\beta$-gal) staining. (B) Flow cytometric analysis of the expression of DcR2 in U2OS cells treated with GSK461364 for $72 \mathrm{~h}$. (C) Quantitative reverse transcription-polymerase chain reaction (qRT-PCR) analysis of interleukin-1 alpha (IL-1 $\alpha$ ) expression relative to that of glyceraldehyde 3-phosphate dehydrogenase (GAPDH) in U2OS cells treated with GSK461364. ${ }^{*} \mathrm{P}<0.05 ;{ }^{* *} \mathrm{P}<0.01$. 
A
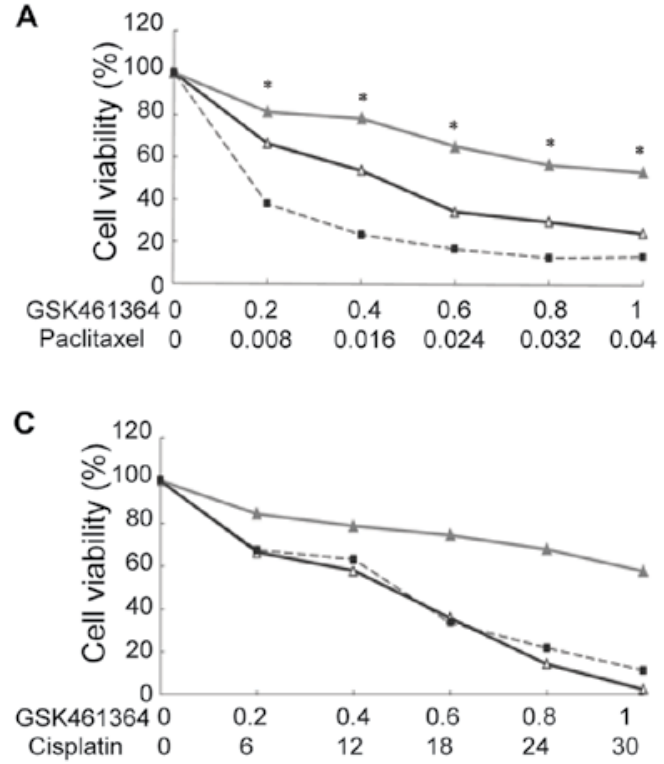

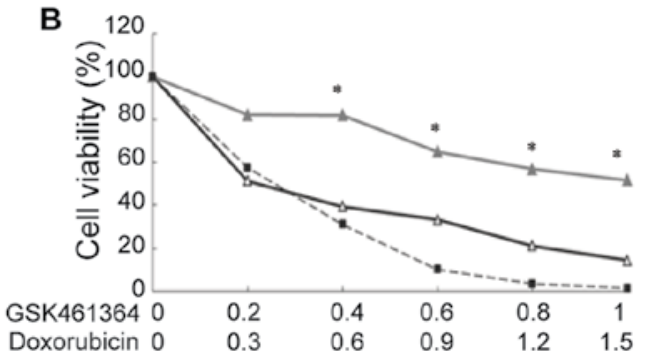

GSK461364

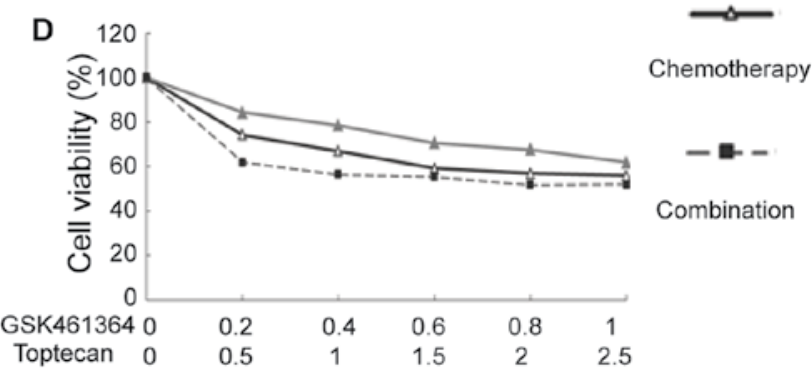

Figure 6. The viability of U2OS cells treated with GSK461364 in combination with different chemotherapeutic agents for 72 h was measured with the TACS ${ }^{\mathrm{TM}}$ MTT cell proliferation assay (expressed as a percentage of viability under controlled culture conditions). All data represent the mean \pm SD of three independent experiments. ${ }^{*}$ A combination index $(\mathrm{CI})<1.0$ indicates a synergistic effect.

A

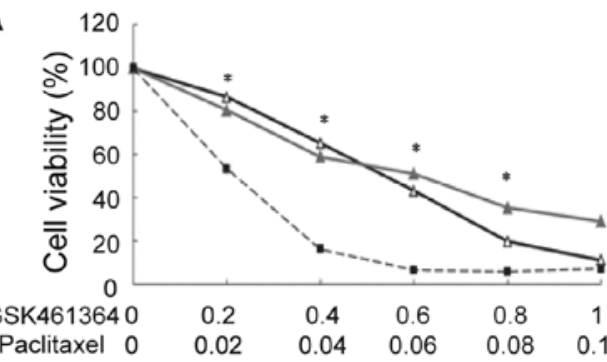

C

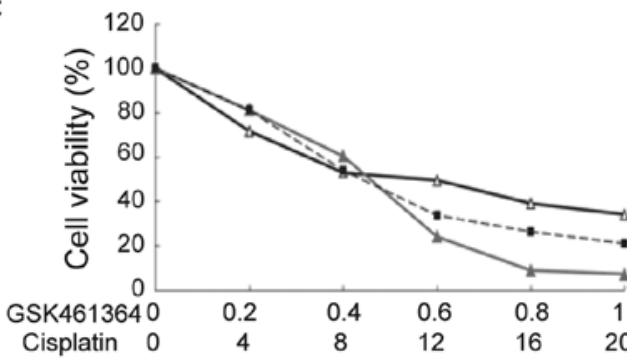

B 120
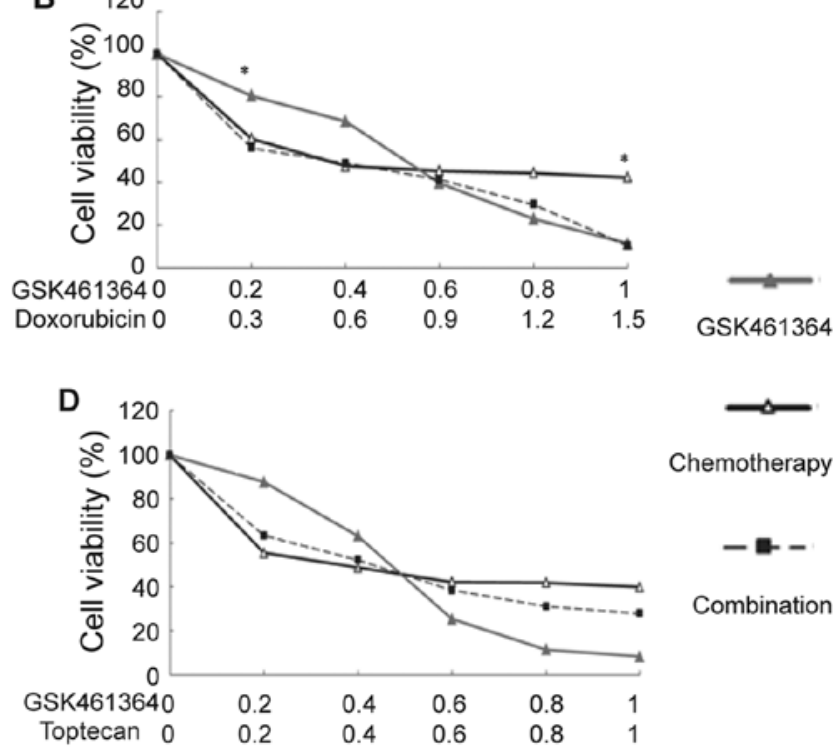

Chemotherapy

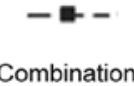

Figure 7. The viability of MG63 cells treated with GSK461364 in combination with different chemotherapeutic agents for $72 \mathrm{~h}$ was measured with the TACS ${ }^{\mathrm{TM}}$ MTT cell proliferation assay (expressed as a percentage of viability under controlled culture conditions). All data represent the mean \pm SD of three independent experiments. *A combination index $(\mathrm{CI})<1.0$ indicates a synergistic effect.

a significant induction of apoptosis in OS cell lines was revealed on co-staining with PI and FITC-labeled Annexin V (Annexin V-FITC; Fig. 4C).

GSK461364 induces cellular senescence in U2OS cell lines. Cellular senescence can be induced by cell cycle inhibition. To explore whether GSK461364 induces senescence in OS cells, a SA- $\beta$-gal assay was used in the U2OS cells following treatment with GSK461364 for $72 \mathrm{~h}$. A significant increase in SA- $\beta$-gal activity in the U2OS cells was revealed following GSK461364 treatment (Fig. 5A). In addition, the expression of DcR2, a well-known senescence biomarker $(34,36)$, was dose-dependently enhanced following GSK461364 administration (Fig. 5B). Furthermore, the expression of IL-1 $\alpha$, a cytokine associated with the senescence-associated secretory phenotype (SASP) $(34,36)$, was upregulated in the U2OS cells treated with GSK461364, as measured through qRT-PCR (Fig. 5C). These results indicate that GSK461364 treatment induces cellular senescence in OS cells.

Synergistic effect of GSK461364 with paclitaxel. We evaluated the possible synergistic effect of GSK461364 with other chemotherapies. As depicted in Figs. 6 and 7, except for the U2OS cells treated with doxorubicin, GSK461364 showed no 


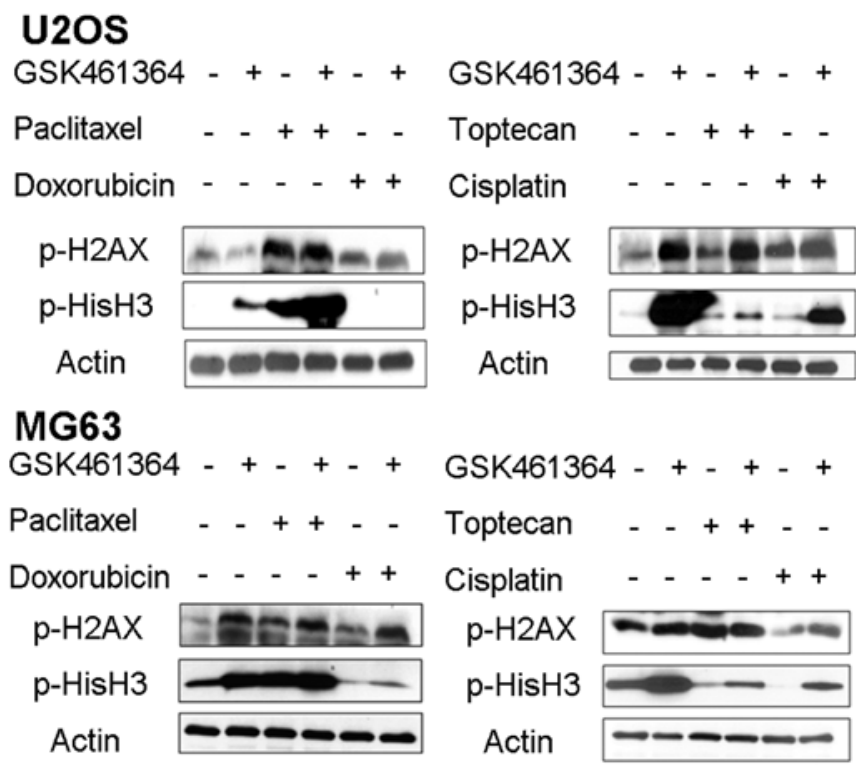

Figure 8. Immunoblotting with antibodies against phospho-Histone H2A.X ( $\mathrm{pH} 2 \mathrm{AX}$ ), phosphorylated histone $\mathrm{H} 3$ (pHisH3), and actin in U2OS (upper panel) and MG63 (lower panel) after being treated with variable combination of GSK461364 and chemotherapy. Only GSK461364 plus paclitaxel showed increased level of $\mathrm{pHisH} 3$ in comparison with other combinations. No significant changes were observed in the $\mathrm{pH} 2 \mathrm{AX}$ level.

significant synergistic effect with DNA damaging agents, such as doxorubicin or cisplatin, or with the topoisomerase inhibitor topotecan. However, it showed a significant synergistic effect with paclitaxel in both U2OS and MG63 cells.

Furthermore, we explored the underlying mechanism of the synergistic effect of GSK461364 and paclitaxel. As depicted in Fig. 8, a combination of GSK461364 and paclitaxel produced significantly increased mitotic arrest, as indicated by the increased pHisH3 level. By contrast, a combination of GSK461364 with other chemotherapeutic agents reduced mitotic arrest. No significant changes were observed in the $\mathrm{pH} 2 \mathrm{AX}$ level, an indicator of DNA damage.

\section{Discussion}

In the present study, we revealed that PLK1 was significantly overexpressed in OS compared with normal osteoblasts or other types of sarcoma. GSK461364, a PLK1 inhibitor, inhibited PLK1 and induced mitotic arrest through G2/M arrest in OS cell lines, with subsequent apoptosis. In addition, GSK461364 induced cellular senescence in OS cell lines and showed a synergistic effect with paclitaxel.

Identifying treatment targets in sarcoma is difficult because of its complex genomic background. However, using genomic and expression profiling in 183 soft tissue sarcoma, Chibon et al (37) established a prognostic gene expression signature, namely, the Complexity index in sarcomas (CINSARC), comprising 67 genes related to mitosis and chromosome management. CINSARC had predicted metastasis outcome in an independent validation set of 127 sarcomas Moreover, by reanalyzing a dataset of GIST, our group identified aurora kinase A (AURKA), along with other cell cycle and mitosis genes, as a prognostic factor for recurrence. In addition, AURKA is a potential treatment target $(38,39)$. Therefore, cell cycle regulation genes are a crucial group of potential biomarkers or targets in sarcoma.

PLK1, essential for regulation of mitosis and maintenance of genomic stability, is overexpressed in human tumors and has prognostic potential in cancers, indicating its involvement in carcinogenesis and its potential as a therapeutic target (22). Sero et al (25) demonstrated enhanced PLK1 expression in clinical OS samples and cell lines compared with normal human tissues. In addition, an enhanced PLK1 expression at diagnosis appeared to be associated with an unfavorable clinical outcome. In the present study, we demonstrated significant PLK1 overexpression in OS by comparing the transcription levels of different subtypes of sarcoma in a microarray database. However, PLK1 was not found to have a prognostic influence in OS (data not shown), and its role in oncogenesis of OS requires further investigation.

Several PLK1 inhibitors have been demonstrated to exert a cytotoxic effect on OS. BI 2536 suppresses cell line growth and clonogenicity (40), and decreases the xenograft tumor size of OS (41). Bogado et al (42) demonstrated that both BI 6727 and GSK461364 induce cell growth arrest, apoptosis and radiosensitization in OS cell lines. Sero et al (25) revealed that another PLK1 inhibitor, NMS-P937, was highly active in both drug-sensitive and drug-resistant OS cell lines.

We revealed that GSK461364 exerts significant cytotoxic activity on 3 OS cell lines. We discovered that GSK461364 targets and downregulates PLK1 and pCDC25C in the OS cell line and induces mitotic arrest, as indicated by an enhanced pHisH3 expression level and a marked G2/M arrest. Our results demonstrated that GSK461364 is cytotoxic to all 3 OS cells when assessed either through an MTT assay or a trypan blue exclusion assay. In addition, a significant induction of apoptosis in OS cell lines was detected by co-staining with PI and Annexin V-FITC, indicating an apoptosis-inducing effect. These data are consistent with previous reports.

Cell cycle inhibitor induces cellular senescence, wherein cells remain viable but typically arrested at the G1 or G2/M phases of the cell cycle, failing to proceed even after mitogen stimulation. Senescence cells are usually characterized by specific cellular phenotypes (such as increased SA- $\beta$-gal activity), secretory phenotype (SASP, usually a cytokine, such as IL-1 $\alpha$ ), and apoptosis-regulatory protein (such as DcR2) (36). In the present study, GSK461364 treatment significantly increased SA- $\beta$-gal activity and enhanced the expression of DcR2 and IL-1 $\alpha$ in OS cell lines. In addition, similar findings were revealed in our previous study of AURKA inhibitor MLN8237 in the treatment of GIST cell line (39). Our studies indicated that cellular senescence is a crucial phenotype of PLK1, or in treatment of other cell cycle inhibitors and that senescence-associated markers may be valuable biomarkers for therapy with these compounds.

Furthermore, GSK461364 and paclitaxel were demonstrated to act synergistically in inducing a cytotoxic effect on OS, probably because of enhanced mitotic arrest. A previous OS study failed to demonstrate any synergistic effect of GSK461364 with chemotherapy (42). However, in a breast cancer study, PLK1-specific antisense oligonucleotides acted synergistically with paclitaxel in inducing cell cycle arrest, apoptosis, and reduction of tumor growth of xenograft (43). 
Therefore, a combination of GSK461364 and paclitaxel deserves further investigation in a clinical setting.

In conclusion, the present study revealed that PLK1 is overexpressed in OS. GSK461364, a PLK1 inhibitor, exerted its cytotoxic effect on OS through the induction of mitotic arrest and subsequent apoptosis and induced cellular senescence; therefore, senescence-associated markers can be used as probable treatment biomarkers, and a combination of GSK461364 and paclitaxel may be effective in OS treatment.

\section{Acknowledgements}

The present study was jointly supported by the grants from the National Science Council (NSC 100-2314-B-075-081 and NSC 101-2314-B-075-029) and the Ministry of Science and Technology, Taiwan (MOST 103-2314-B-075-066), the Taipei Veterans General Hospital (V102E8-003, V103E8-001, V101C-133, V102C-034, V103C-188, V104C-099, V104E16001-MY3-1) and from the Yen Tjing Ling Medical Foundation (grant number CI-100-19 and CI-103-6) designated to Dr Chueh-Chuan Yen. This study was also supported by the Taiwan Clinical Oncology Research Foundation, and the Chong Hin Loon Memorial Cancer and Biotherapy Research Center of National Yang-Ming University.

\section{References}

1. Hung GY, Horng JL, Yen HJ, Yen CC, Chen WM, Chen PC, Wu HT and Chiou HJ: Incidence patterns of primary bone cancer in Taiwan (2003-2010): A population-based study. Ann Surg Oncol 21: 2490-2498, 2014.

2. Siegel R, Naishadham D and Jemal A: Cancer statistics, 2012. CA Cancer J Clin 62: 10-29, 2012.

3. Hung GY, Yen HJ, Yen CC, Chen WM, Chen PC, Wu HT, Chiou HJ, Chang WH and Hsu HE: Experience of pediatric osteosarcoma of the extremity at a single institution in Taiwan: Prognostic factors and impact on survival. Ann Surg Oncol 22: 1080-1087, 2015.

4. Ferrari S, Smeland S, Mercuri M, Bertoni F, Longhi A, Ruggieri P, Alvegard TA, Picci P, Capanna R, Bernini G, et al; Italian and Scandinavian Sarcoma Groups: Neoadjuvant chemotherapy with high-dose Ifosfamide, high-dose methotrexate, cisplatin, and doxorubicin for patients with localized osteosarcoma of the extremity: A joint study by the Italian and Scandinavian Sarcoma Groups. J Clin Oncol 23: 8845-8852, 2005.

5. Iwamoto Y, Tanaka K, Isu K, Kawai A, Tatezaki S, Ishii T, Kushida K, Beppu Y, Usui M, Tateishi A, et al: Multiinstitutional phase II study of neoadjuvant chemotherapy for osteosarcoma (NECO study) in Japan: NECO-93J and NECO-95J. J Orthop Sci 14: 397-404, 2009

6. Harting MT, Lally KP, Andrassy RJ, Vaporciyan AA, Cox CS Jr, Hayes-Jordan A and Blakely ML: Age as a prognostic factor for patients with osteosarcoma: An analysis of 438 patients. J Cancer Res Clin Oncol 136: 561-570, 2010.

7. Joo MW, Shin SH, Kang YK, Kawai A, Kim HS, Asavamongkolkul A, Jeon DG, Kim JD, Niu X, Tsuchiya H, et al: Osteosarcoma in Asian populations over the age of 40 years: A Multicenter study. Ann Surg Oncol 22: 3557-3564, 2015.

8. Janeway KA, Barkauskas DA, Krailo MD, Meyers PA, Schwartz CL, Ebb DH, Seibel NL, Grier HE, Gorlick R and Marina N: Outcome for adolescent and young adult patients with osteosarcoma: A report from the Children's Oncology Group. Cancer 118: 4597-4605, 2012.

9. Whelan JS, Jinks RC, McTiernan A, Sydes MR, Hook JM, Trani L, Uscinska B, Bramwell V, Lewis IJ, Nooij MA, et al: Survival from high-grade localised extremity osteosarcoma: Combined results and prognostic factors from three European Osteosarcoma Intergroup randomised controlled trials. Ann Oncol 23: 1607-1616, 2012.
10. Mejia-Guerrero S, Quejada M, Gokgoz N, Gill M, Parkes RK, Wunder JS and Andrulis IL: Characterization of the 12q15 MDM2 and 12q13-14 CDK4 amplicons and clinical correlations in osteosarcoma. Genes Chromosomes Cancer 49: 518-525, 2010.

11. Crago AM, Socci ND, DeCarolis P, O'Connor R, Taylor BS, Qin LX, Antonescu CR and Singer S: Copy number losses define subgroups of dedifferentiated liposarcoma with poor prognosis and genomic instability. Clin Cancer Res 18: 1334-1340, 2012.

12. Wunder JS, Nielsen TO, Maki RG, O'Sullivan B and Alman BA: Opportunities for improving the therapeutic ratio for patients with sarcoma. Lancet Oncol 8: 513-524, 2007.

13. Ladanyi M, Cha C, Lewis R, Jhanwar SC, Huvos AG and Healey JH: MDM2 gene amplification in metastatic osteosarcoma. Cancer Res 53: 16-18, 1993.

14. Koshkina NV, Kleinerman ES, Li G, Zhao CC, Wei Q and Sturgis EM: Exploratory analysis of Fas gene polymorphisms in pediatric osteosarcoma patients. J Pediatr Hematol Oncol 29: 815-821, 2007.

15. Choy E, Hornicek F, MacConaill L, Harmon D, Tariq Z, Garraway L and Duan Z: High-throughput genotyping in osteosarcoma identifies multiple mutations in phosphoinositide3-kinase and other oncogenes. Cancer 118: 2905-2914, 2012.

16. Perry JA, Kiezun A, Tonzi P, Van Allen EM, Carter SL, Baca SC, Cowley GS, Bhatt AS, Rheinbay E, Pedamallu CS, et al: Complementary genomic approaches highlight the PI3K/mTOR pathway as a common vulnerability in osteosarcoma. Proc Natl Acad Sci USA 111: E5564-E5573, 2014.

17. Scheel C, Schaefer KL, Jauch A, Keller M, WaiD, BrinkschmidtC, van Valen F, Boecker W, Dockhorn-Dworniczak B and Poremba C: Alternative lengthening of telomeres is associated with chromosomal instability in osteosarcomas. Oncogene 20: 3835-3844, 2001.

18. Wan X, Mendoza A, Khanna C and Helman LJ: Rapamycin inhibits ezrin-mediated metastatic behavior in a murine model of osteosarcoma. Cancer Res 65: 2406-2411, 2005.

19. Yen CC, Chen WM, Chen TH, Chen WY, Chen PC, Chiou HJ, Hung GY, Wu HT, Wei CJ, Shiau CY, et al: Identification of chromosomal aberrations associated with disease progression and a novel 3q13.31 deletion involving LSAMP gene in osteosarcoma. Int J Oncol 35: 775-788, 2009.

20. Pasic I, Shlien A, Durbin AD, Stavropoulos DJ, Baskin B, Ray PN, Novokmet A and Malkin D: Recurrent focal copynumber changes and loss of heterozygosity implicate two noncoding RNAs and one tumor suppressor gene at chromosome 3q13.31 in osteosarcoma. Cancer Res 70: 160-171, 2010.

21. Grignani G, Palmerini E, Ferraresi V, D'Ambrosio L, Bertulli R, Asaftei SD, Tamburini A, Pignochino Y, Sangiolo D, Marchesi E, et al; Italian Sarcoma Group: Sorafenib and everolimus for patients with unresectable high-grade osteosarcoma progressing after standard treatment: A non-randomised phase 2 clinical trial. Lancet Oncol 16: 98-107, 2015.

22. Strebhardt $K$ and Ullrich A: Targeting polo-like kinase 1 for cancer therapy. Nat Rev Cancer 6: 321-330, 2006.

23. Duan Z, Ji D, Weinstein EJ, Liu X, Susa M, Choy E, Yang C, Mankin H and Hornicek FJ: Lentiviral shRNA screen of human kinases identifies PLK1 as a potential therapeutic target for osteosarcoma. Cancer Lett 293: 220-229, 2010.

24. Yamaguchi U, Honda K, Satow R, Kobayashi E, Nakayama R, Ichikawa H, Shoji A, Shitashige M, Masuda M, Kawai A, et al: Functional genome screen for therapeutic targets of osteosarcoma. Cancer Sci 100: 2268-2274, 2009.

25. Sero V, Tavanti E, Vella S, Hattinger CM, Fanelli M, Michelacci F, Versteeg R, Valsasina B, Gudeman B, Picci P, et al: Targeting polo-like kinase 1 by NMS-P937 in osteosarcoma cell lines inhibits tumor cell growth and partially overcomes drug resistance. Invest New Drugs 32: 1167-1180, 2014.

26. Yen CC, Hsiao CD, Chen WM, Wen YS, Lin YC, Chang TW, Yao FY, Hung SC, Wang JY, Chiu JH, et al: Cytotoxic effects of 15d-PGJ2 against osteosarcoma through ROS-mediated AKT and cell cycle inhibition. Oncotarget 5: 716-725, 2014.

27. Gilmartin AG, Bleam MR, Richter MC, Erskine SG, Kruger RG, Madden L, Hassler DF, Smith GK, Gontarek RR, Courtney MP, et al: Distinct concentration-dependent effects of the polo-like kinase 1-specific inhibitor GSK461364A, including differential effect on apoptosis. Cancer Res 69: 6969-6977, 2009. 
28. Li C and Hung Wong W: Model-based analysis of oligonucleotide arrays: model validation, design issues and standard error application. Genome Biol 2: Research0032, 2001.

29. Li C and Wong WH: Model-based analysis of oligonucleotide arrays: Expression index computation and outlier detection. Proc Natl Acad Sci USA 98: 31-36, 2001.

30. Mosmann T: Rapid colorimetric assay for cellular growth and survival: Application to proliferation and cytotoxicity assays. J Immunol Methods 65: 55-63, 1983.

31. Chou TC and Talalay P: Quantitative analysis of dose-effect relationships: The combined effects of multiple drugs or enzyme inhibitors. Adv Enzyme Regul 22: 27-55, 1984.

32. Schmit TL, Nihal M, Ndiaye M, Setaluri V, Spiegelman VS and Ahmad N: Numb regulates stability and localization of the mitotic kinase PLK1 and is required for transit through mitosis. Cancer Res 72: 3864-3872, 2012.

33. Tuveson DA, Willis NA, Jacks T, Griffin JD, Singer S, Fletcher CD, Fletcher JA and Demetri GD: STI571 inactivation of the gastrointestinal stromal tumor c-KIT oncoprotein Biological and clinical implications. Oncogene 20: 5054-5058, 2001.

34. Zhu Y, Xu L, Zhang J, Hu X, Liu Y, Yin H, Lv T, Zhang H, Liu L, An H, et al: Sunitinib induces cellular senescence via p53/ Dec1 activation in renal cell carcinoma cells. Cancer Sci 104: 1052-1061, 2013

35. Müller-Tidow C, Metzger R, Kügler K, Diederichs S, Idos G, Thomas M, Dockhorn-Dworniczak B, Schneider PM, Koeffler HP, Berdel WE, et al: Cyclin E is the only cyclindependent kinase 2-associated cyclin that predicts metastasis and survival in early stage non-small cell lung cancer. Cancer Res 61: 647-653, 2001.

36. Ewald JA, Desotelle JA, Wilding G and Jarrard DF: Therapyinduced senescence in cancer. J Natl Cancer Inst 102: 1536-1546, 2010.
37. Chibon F, Lagarde P, Salas S, Pérot G, Brouste V, Tirode F, Lucchesi C, de Reynies A, Kauffmann A, Bui B, et al: Validated prediction of clinical outcome in sarcomas and multiple types of cancer on the basis of a gene expression signature related to genome complexity. Nat Med 16: 781-787, 2010.

38. Yen CC, Yeh CN, Cheng CT, Jung SM, Huang SC, Chang TW, Jan YY, Tzeng CH, Chao TC, Chen YY, et al: Integrating bioinformatics and clinicopathological research of gastrointestinal stromal tumors: Identification of aurora kinase A as a poor risk marker. Ann Surg Oncol 19: 3491-3499, 2012.

39. Yeh CN, Yen CC, Chen YY, Cheng CT, Huang SC, Chang TW, Yao FY, Lin YC, Wen YS, Chiang KC, et al: Identification of aurora kinase $\mathrm{A}$ as an unfavorable prognostic factor and potential treatment target for metastatic gastrointestinal stromal tumors. Oncotarget 5: 4071-4086, 2014.

40. Morales AG, Brassesco MS, Pezuk JA, Oliveira JC, Montaldi AP, Sakamoto-Hojo ET, Scrideli CA and Tone LG: BI 2536-mediated PLK1 inhibition suppresses HOS and MG-63 osteosarcoma cell line growth and clonogenicity. Anticancer Drugs 22: 995-1001, 2011.

41. Liu X, Choy E, Harmon D, Yang S, Yang C, Mankin H, Hornicek FJ and Duan Z: Inhibition of polo-like kinase 1 leads to the suppression of osteosarcoma cell growth in vitro and in vivo. Anticancer Drugs 22: 444-453, 2011.

42. Bogado RF, Pezuk JA, de Oliveira HF, Tone LG and Brassesco MS: BI 6727 and GSK461364 suppress growth and radiosensitize osteosarcoma cells, but show limited cytotoxic effects when combined with conventional treatments. Anticancer Drugs 26: 56-63, 2015.

43. Spankuch B, Heim S, Kurunci-Csacsko E, Lindenau C, Yuan J, Kaufmann M and Strebhardt K: Down-regulation of Polo-like kinase 1 elevates drug sensitivity of breast cancer cells in vitro and in vivo. Cancer Res 66: 5836-5846, 2006. 